\title{
Regulation of membrane type-1 matrix metalloproteinase activity and intracellular localization in clinical thoracic aortic aneurysms
}

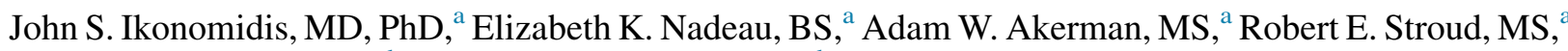
Rupak Mukherjee, $\mathrm{PhD},{ }^{\mathrm{a}, \mathrm{b}}$ and Jeffrey A. Jones, $\mathrm{PhD}^{\mathrm{a}, \mathrm{b}}$

\section{ABSTRACT}

Objective: Membrane type-1 matrix metalloproteinase (MT1-MMP) is elevated during thoracic aortic aneurysm (TAA) development in mouse models, and plays an important role in the activation of matrix metalloproteinase (MMP)- 2 and the release of matrix- bound transforming growth factor- $\beta$. In this study, we tested the hypothesis that MT1-MMP is subject to protein kinase C (PKC)-mediated regulation, which alters intracellular trafficking and activity with TAAs.

Methods: Levels of MMP-2, native and phosphorylated MT1-MMP, and PKC- $\delta$ were measured in aortic tissue from patients with small TAAs $(<5 \mathrm{~cm} ; \mathrm{n}=8)$ and large TAAs $(>6.5 \mathrm{~cm} ; \mathrm{n}=8)$, and compared with values measured in normal controls $(\mathrm{n}=8)$. Cellular localization of green fluorescent protein (GFP)-tagged MT1-MMP was assessed in aortic fibroblasts isolated from control and 4-week TAA mice. The effects of PKC-mediated phosphorylation on MT1-MMP cellular localization and function (active MMP-2 vs phospo-Smad2 abundance) were assessed after treatment with a PKC activator (phorbol-12-myristate-13-acetate [PMA], $100 \mathrm{nM}$ ) with and without a PKC- $\delta$-specific inhibitor (röttlerin, $3 \mu \mathrm{M}$ ).

Results: Compared with controls, MT1-MMP abundance was increased in aortas from both TAA groups. Active MMP-2 was increased only in the large TAA group. The abundances of phosphorylated MT1-MMP and activated PKC- $\delta$ were enhanced in the small TAA group compared with the large TAA group. MT1-MMP was localized on the plasma membrane in aortic fibroblasts from control mice and in endosomes from TAA mice. Treatment with PMA induced MT1-MMP-GFP internalization, enhanced phospho-Smad2, and reduced MMP-2 activation, whereas röttlerin pretreatment inhibited these effects.

Conclusions: Phosphorylation of MT1-MMP mediates its activity through directing cellular localization, shifting its role from MMP-2 activation to intracellular signaling. Thus, targeted inhibition of MT1-MMP may have therapeutic relevance as an approach to attenuating TAA development. (J Thorac Cardiovasc Surg 2017;153:537-46)

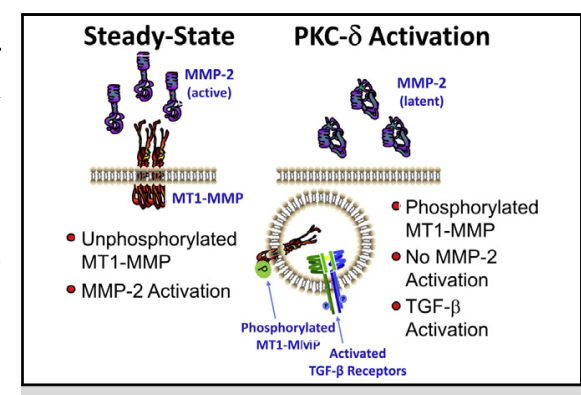

PKC- $\delta$-mediated phosphorylation of MT1-MMP regulates its intracellular localization.

\section{Central Message}

Targeting MT1-MMP activity or protein abundance may provide a novel therapeutic strategy for treating TAAs.

\section{Perspective}

Previous studies have identified and characterized a multifunctional membrane-bound protease, MT1-MMP, with several unique characteristics that position it at the center of multiple mechanisms capable of regulating both ECM deposition and degradation. Phosphorylation of MT1-MMP regulates its cellular localization, shifting its functional role from pericellular proteolysis to intracellular signaling.

See Editorial Commentary page 547

\footnotetext{
From the ${ }^{\mathrm{a}}$ Division of Cardiothoracic Surgery, Medical University of South Carolina; and ${ }^{\mathrm{b}}$ Research Service, Ralph H. Johnson Veterans Affairs Medical Center, Charleston, SC.

This work was supported by grants from the National Institutes of Health (NHLBI R01 HL102121, to J.S.I.) and the Department of Veterans Affairs (VA-ORD BLR\&D Merit I01BX000904, to J.A.J.).

Read at the 42nd Annual Meeting of The Western Thoracic Surgical Association, Waikoloa, Hawaii, June 22-25, 2016.

Received for publication June 21, 2016; revisions received Sept 19, 2016; accepted for publication Oct 4, 2016; available ahead of print Dec 4, 2016.

Address for reprints: Jeffrey A. Jones, PhD, Division of Cardiothoracic Surgery Research, Medical University of South Carolina, Strom Thurmond Research Building, 114 Doughty St, Suite 338, MSC778, Charleston, SC 29425 (E-mail: jonesja@musc.edu).

$0022-5223 / \$ 36.00$

Copyright (C) 2016 by The American Association for Thoracic Surgery

http://dx.doi.org/10.1016/j.jtcvs.2016.10.065
}

Thoracic aortic aneurysms (TAAs) constitute a potentially deadly disease that carries significant morbidity and mortality and develops in the absence of overt symptomology. ${ }^{1}$ It is now well understood that TAAs form in response to dysregulated remodeling of the aortic extracellular matrix

Scanning this QR code will take you to the article title page.

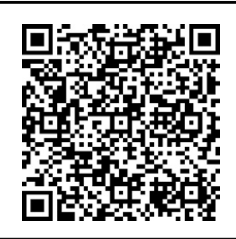




$$
\begin{array}{ll}
\text { Abbreviations and Acronyms } \\
\begin{aligned}
\text { ECM } & =\text { extracellular matrix } \\
\text { GFP } & =\text { green fluorescent protein } \\
\text { MMP } & =\text { matrix metalloproteinase } \\
\text { MT1-MMP }= & \text { membrane type-1 matrix } \\
& \text { metalloproteinase } \\
\text { PBS } & =\text { phosphate-buffered saline } \\
\text { PMA } & =\text { phorbol 12-myristate 13-acetate } \\
\text { PKC } & =\text { protein kinase C } \\
\text { TAA } & =\text { thoracic aortic aneurysm } \\
\text { TGF } & =\text { transforming growth factor }
\end{aligned}
\end{array}
$$

(ECM), tipping the homeostatic balance between matrix deposition and degradation in favor of proteolysis, with subsequent fragmentation of the elastic lamellae and remodeling of the collagen matrix. These processes are mediated in part by a family of endopeptidases, the matrix metalloproteinases (MMPs). ${ }^{2}$ Key members of this family, including MMP-2 and membrane type 1 MMP (MT1-MMP), play well-described roles in aneurysm development in both the abdomen and the thorax. ${ }^{3-6}$ Furthermore, substantial evidence implicates altered transforming growth factor (TGF) $-\beta$ signaling as a primary contributor to structural remodeling. . $^{5,8}$ The molecular mechanisms underlying the regulation of these processes remain elusive, however., ${ }^{9,10}$

Previous studies from this laboratory and others have demonstrated increased MT1-MMP abundance in both human specimens and animal models of TAA, suggesting that this enzyme plays an important role in mediating the pathological remodeling process. ${ }^{3,6,11,12}$ Several unique characteristics of MT1-MMP contribute to its prescribed role as a master regulator of matrix remodeling. First, MT1MMP has a diverse portfolio of proteolytic substrates and plays an integral role in mediating pericellular proteolysis at the cell-matrix interface. ${ }^{3,13-16}$ Second, MT1-MMP can activate other MMPs, such as pro-MMP-2 and pro-MMP-13, ${ }^{17,18}$ thereby amplifying its proteolytic response. Third, MT1MMP also plays an important regulatory role in releasing ECM-sequestered matrikines, cytokines, and growth factors, such as TGF- $\beta .^{14,19}$ Finally, as a type I transmembrane protein, MT1-MMP cell surface expression has been shown to be regulated through an endosomal recycling process mediated by phosphorylation. ${ }^{20-23}$ Importantly, previous studies have suggested that MT1-MMP is phosphorylated at amino acid threonine-567 by protein kinase C (PKC). ${ }^{24,25}$

Together, these unique features position MT1-MMP as a key mediator capable of regulating aortic structural remodeling through multiple pathways. Importantly, understanding the mechanisms governing MT1-MMP cellular function may contribute to the identification of therapeutic strategies for treating aneurysm disease. In the present study, we tested the hypothesis that MT1-MMP activity and function in TAA disease are regulated by phosphorylation.

\section{METHODS \\ Study Population}

Based on previous results using an established murine model of calcium chloride-induced TAA, MT1-MMP protein abundance was elevated in TAA-induced mice, and a positive correlation between MT1-MMP activity and the time-dependent change in aortic diameter was identified. ${ }^{3}$ With this information in mind, human ascending aortic tissue specimens $(n=16)$ were selected from a multi-institutional tissue bank, based on size intervals that bracketed the full spectrum of sizes present in the tissue bank, the rationale being that the small aneurysm specimens represented those occurring earlier in aneurysm development, whereas the large aneurysm specimens were effectively closer to end stage. The specimens were then stratified into 2 groups for analysis: small TAAs $(4.0-4.9 \mathrm{~cm} ; \mathrm{n}=8)$ and large TAAs $(6.5-8.0 \mathrm{~cm} ; \mathrm{n}=8)$. All aortic tissue specimens were obtained from patients with idiopathic degenerative aortic aneurysms with tricuspid aortic valves undergoing root replacement. All specimens were collected from the widest region of the ascending aorta; no specimens were taken from the aortic sinus or proximal arch. No patients had aortic dissection, inflammatory aortic disease, or a known syndromic aortic disease, such as Marfan syndrome, Ehlers-Danlos syndrome, or Loeys-Dietz syndrome. Care was taken to ensure that all tissue specimens were harvested from the same region of the ascending aorta. Normal aortic specimens were harvested from heart transplant donors or recipients $(n=8)$ with no known aortic disease. This study was approved by the Institutional Review Boards of the Medical University of South Carolina and University of Pennsylvania. Informed consent was obtained from all patients.

In addition, primary mouse aortic cell lines were isolated $(n=6$ independently derived, descending aortic fibroblast cell lines from normal mice; $\mathrm{n}=4$ independently derived descending aortic fibroblast cell lines isolated from mice at 4 weeks after TAA induction) and were used to examine the regulation of MT1-MMP and its functional outcomes in passages 2 to 7 . TAAs were induced as described previously. ${ }^{3,8,26}$

\section{Aortic Sample Preparation}

Immediately after resection, aortic tissue specimens (both human and mouse) were snap-frozen in dry ice/ethanol slurry and then stored at $-80^{\circ} \mathrm{C}$. Upon thawing, the specimens were transferred to cold extraction/homogenization buffer (buffer volume 1:6 wt/vol) containing $10 \mathrm{mM}$ cacodylic acid $\mathrm{pH} 5.0,0.15 \mathrm{M} \mathrm{NaCl}, 10 \mathrm{mM} \mathrm{ZnCl} 2,1.5 \mathrm{mM}$ $\mathrm{NaN}_{3}$, and $0.01 \%$ Triton $\mathrm{X}-100$ (vol/vol), and disrupted in a bead mill homogenizer (Tissuelyser; Qiagen, Valencia, Calif). Homogenates were centrifuged $\left(800 \times g\right.$ for 10 minutes at $\left.4^{\circ} \mathrm{C}\right)$, and the supernatants were used for immunoblotting and gelatin zymography.

\section{Immunoblot Analysis}

For immunoblotting, $10 \mu \mathrm{g}$ of each aortic homogenate was loaded on a $4 \%$ to $12 \%$ bis-tris gradient gel and fractionated by electrophoresis. The proteins were transferred to nitrocellulose membranes $(0.45 \mu \mathrm{m}$; Bio-Rad, Hercules, Calif) and incubated in antisera specific for MT1-MMP (0.4 $\mu \mathrm{g} / \mathrm{mL}$; AB815; EMD Millipore, Temecula, Calif), MMP-2 (1:2000; 04-1048; EMD Millipore), MT1-MMP (1:2000; ab38971; Abcam, Cambridge, Mass), phospho-threonine (1:1000; sc-5267, Santa Cruz Biotechnology, Dallas, Tex), PKC- $\delta$ (1:1000; sc-937; Santa Cruz Biotechnology), phospho-Smad2 (1:2000; 3104; Cell Signaling Technology, Beverly, Mass), and phospho-PKC- $\delta$ (1:1000; 9374S, Cell Signaling Technology) were diluted into $5 \%$ nonfat dry milk/phosphatebuffered saline (PBS). A secondary peroxidase-conjugated antibody (primary antibody species-specific) was applied (1:5000; 5\% nonfat dry milk/PBS), and signals were detected with a chemiluminescent substrate (Western Lighting Chemiluminescence Reagent Plus; PerkinElmer, San 
Jose, Calif) and recorded on film. Band intensity was quantified using Gel-Pro Analyzer version 3.1.14 (Media Cybernetics, Silver Spring, Md).

\section{Gelatin Zymography}

Abundance of MMP-2 was assessed by gelatin zymography. ${ }^{12}$ Homogenates (10 $\mu \mathrm{g}$ protein) were fractionated on a nondenaturing $10 \%$ polyacrylamide gel containing $0.1 \%(\mathrm{wt} / \mathrm{vol})$ gelatin (Invitrogen, Carlsbad, Calif). The gels were then equilibrated and incubated in Zymogram developing buffer (Invitrogen) for 18 hours at $37^{\circ} \mathrm{C}$. After staining with $0.5 \%$ Coomassie brilliant blue for 2 hours at room temperature, the gels were destained to reveal regions of gelatin clearance. The relative abundance of MMP-2 (as verified by a recombinant MMP-2 standard) was then determined by densitometry using the Gel-Pro Analyzer software.

\section{Immunoprecipitation}

In brief, $0.15 \mathrm{~g}$ of aortic tissue was suspended in $1.5 \mathrm{~mL}$ of standard RIPA buffer $(50 \mathrm{mM}$ Tris-HCl pH 7.4, $150 \mathrm{mM} \mathrm{NaCl}, 1 \mathrm{mM}$ phenylmethylsulfonyl fluoride, $1 \mathrm{mM}$ ethylenediaminetetraacetic acid, $5 \mu \mathrm{g} / \mathrm{mL}$ aprotinin, $5 \mu \mathrm{g} / \mathrm{mL}$ leupeptin, $1 \%$ Triton $\mathrm{X}-100,1 \%$ sodium deoxycholate, and $0.1 \%$ sodium dodecyl sulfate) and disrupted using a bead mill homogenizer. The homogenate was centrifuged $(800 \times g$ for 10 minutes at $4^{\circ} \mathrm{C}$ ), and a Pierce BCA protein assay was completed (23225; Thermo Fisher Scientific). For this, $500 \mu \mathrm{g}$ of total protein was mixed with $5.0 \mu \mathrm{g}$ of MT1-MMP antibody (Abcam; ab38971) and allowed to incubate overnight at $4{ }^{\circ} \mathrm{C}$ with gentle agitation. Subsequently, $50 \mu \mathrm{L}$ of equilibrated (RIPA buffer) Protein A/G Plus-Agarose (Santa Cruz Biotechnology; sc-2003) was added, followed by a 1-hour incubation at $4^{\circ} \mathrm{C}$ with gentle agitation. Immunoprecipitates were collected by centrifugation at $1500 \times g$ for 5 minutes at $4^{\circ} \mathrm{C}$, after which the pellets were washed four times with PBS. The final pellet was resuspended in $60 \mu \mathrm{L}$ of $2 \times$ Laemmli sample buffer and boiled for 5 minutes. The precipitated proteins were fractionated on $4 \%$ to $12 \%$ bis-tris gradient polyacrylamide gels, and phospho-threonine phosphorylated MT1-MMP was measured by Western blot analysis.

\section{PKC Activation/Inhibition and Confocal Microscopy}

Primary aortic fibroblasts from normal (control) or 4-week TAA-induced mice were plated in glass-bottom dishes and transfected with $1.5 \mu \mathrm{g}$ of pCMV-MT1-MMP-GFP (C-terminal tag; green fluorescence protein [GFP]) using Lipofectamine 2000 (Thermo Fisher Scientific, Waltham, Mass) according to the manufacturer's instructions. Cells were allowed to grow for 48 hours and were then fixed with $3.7 \%$ paraformaldehyde at $37^{\circ} \mathrm{C}$. Nuclei were counterstained with 4',6-diamidino-2-phenylindole (blue fluorescence). In addition, normal aortic fibroblasts, grown in either plastic (biochemistry)- or glass (microscopy)-bottom dishes, were transfected with CMV-MT1-MMPGFP and allowed to grow for 48 hours. The cells were then treated, in the absence (vehicle control, dimethyl sulfoxide, 1:1000 dilution) or presence of phorbol 12-myristate 13-acetate (PMA), $100 \mathrm{nM}$, for 30 minutes at $37^{\circ} \mathrm{C}$, with or without the PKC- $\delta$-specific inhibitor röttlerin, $3 \mu \mathrm{M}$, for a 3-hour preincubation plus 30 minutes of concurrent exposure at $37^{\circ} \mathrm{C}$. The cells were either collected for immunoblotting for phospho-Smad 2 or prefixed (10 minutes in $1.5 \%$ paraformaldehyde, diluted $2 \times$ into the growth medium), followed by normal fixation (10 minutes in $3.7 \%$ paraformaldehyde at $37^{\circ} \mathrm{C}$ ) to preserve membrane localization. A second cohort of cells was treated longer (18 hours) with PMA or vehicle, with or without röttlerin, to determine changes in protein content (100 nM PMA $\pm 3 \mu \mathrm{M}$ röttlerin for 3 hours, followed by a 15-hour incubation in fresh drug-free medium). These cells were collected for immunoblotting for active and latent MMP-2, as well as phospho-Smad2. All cell images were captured using an Inverted Nikon A1R + confocal microscope, with a $60 \times 1.5$ NA oil emersion objective. Representative images of at least 3 experiments are shown.

\section{Data Analysis}

Relative protein abundances of MT1-MMP, MMP-2 (active and total forms), PKC- $\delta$, phospho-PKC- $\delta$, and phospho-Smad 2 were determined from densitometry of films or zymographic gels. Integrated optical densities were determined for each specimen, and the results were expressed as the mean percentage change of normal aorta. Before statistical analysis, the Shapiro-Wilk test was performed on the original data to determine normality. Differences in patient age and differences in murine MT1-MMP abundance were compared using the unpaired 2-sample $t$ test. For all analyses that were not normally distributed, a log transformation was performed on the original data, and normality was confirmed. Using intercooled Stata 8.2 (StataCorp, College Station, Tex), the normally distributed data were then analyzed by one-way ANOVA, with Tukey's wholly significant difference (WSD) post hoc analysis for the separation of means. All results demonstrating a $P$ value $<.05$ were considered statistically significant. The original data (nontransformed) were then graphically represented using box-and-whisker plots, including both median and mean values as different colored bars within the box, which depicts the 25 th to 75 th interquartile range. In addition, each individual data point is represented as an independent dot overlaying the box.

\section{RESULTS}

Patient demographic information is summarized in Table 1. Patients with large TAAs were older than those with small TAAs, and patients in both TAA groups were older than the normal referent control patients.

Protein abundance of MT1-MMP was significantly increased in small TAAs $(168 \% \pm 20 \% ; P<.05$ vs normal $)$ and large TAAs $(206 \% \pm 37 \% ; P<.05$ vs normal), compared with normal aorta $(100 \% \pm 10 \%)$ (Figure 1 , A). The abundance of the $64-\mathrm{kDa}$ active form of MMP-2 was elevated in the large TAAs only $(273 \% \pm 70 \%$; $P<.05$ vs normal, $P<.05$ vs small TAA) compared with normal aorta $(100 \% \pm 40 \%)$ and small TAA $(117 \% \pm 31 \%)$ (Figure $1, B)$. No change in TIMP-2 abundance compared with normal aorta was observed in either the small TAA or large TAA group (data not shown).

Protein homogenates from the normal, small TAA, and large TAA groups were then subjected to MT1-MMP-specific immunoprecipitation. The resulting immunoprecipitated proteins were then fractionated by gel electrophoresis and immunoblotted for MT1-MMP (to verify functional immunoprecipitation), and with an anti-phospho-threonine antibody. The results show increased phospho-MT1-MMP in both the small TAA $(973 \% \pm 272 \% ; P<.05$ vs normal) and large TAA $(410 \% \pm 80 \% ; P<.05$ vs normal) groups compared

TABLE 1. Patient demographic data

\begin{tabular}{lccc}
\hline \multicolumn{1}{c}{ Group } & Number & Age, $\mathbf{y}$, mean \pm SEM & Size, $\mathbf{c m}$, range \\
\hline Normal & 8 & $45.3 \pm 5.0^{*}$ & $2.9-3.2$ \\
Small TAA & 8 & $59.7 \pm 4.0^{*}$ & $4.0-4.9$ \\
Large TAA & 8 & $73.3 \pm 4.0^{*}, \dagger$ & $6.5-8.0$ \\
\hline
\end{tabular}

SEM, Standard error of the mean; TAA, thoracic aortic aneurysm. $* P<.05$ versus normal. $\dagger P<.05$ versus small TAA. 

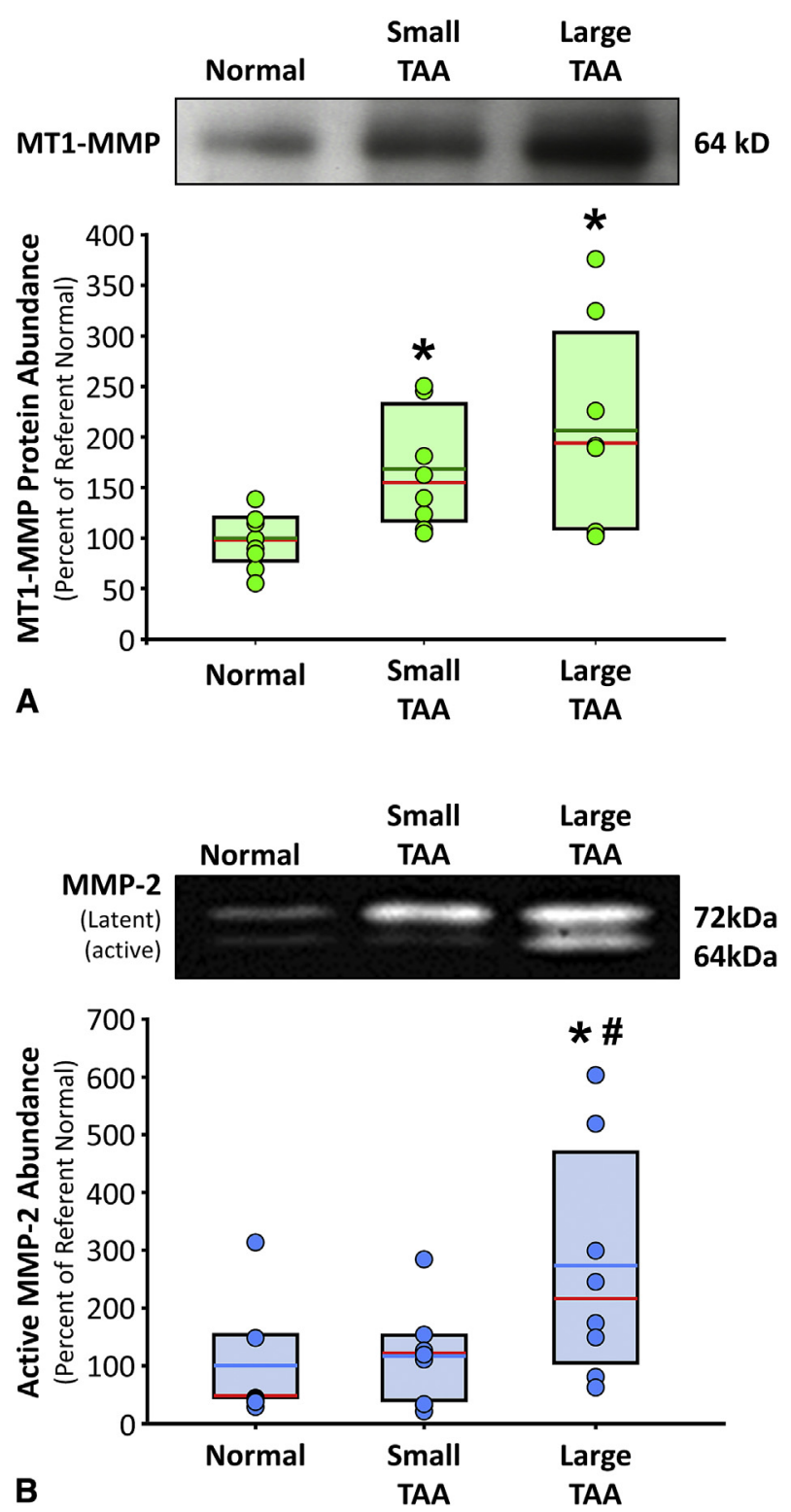

FIGURE 1. MT1-MMP and MMP-2 abundance in clinical TAA specimens. (A) (Top) Representative immunoblot showing MT1-MMP protein abundance in the normal aorta $(\mathrm{n}=8)$, small TAA $(4.0-4.9 \mathrm{~cm}$; $\mathrm{n}=8)$, and large TAA $(6.5-8.0 \mathrm{~cm} ; \mathrm{n}=8)$ groups. (Bottom) Densitometric quantitation of MT1-MMP protein abundance determined by immunoblotting. Results are expressed as mean percentage change of normal aorta. Individual data points in each group overlay box plots. The box defines the 25 th to 75 th interquartile range, and both the median (red line) and mean (green line) values are shown within the box. $* P<.05$ versus normal, ANOVA with Tukey's WSD test. (B) (Top) Representative zymogram showing the protein abundance of the latent form (72-kDa band) and active form (64-kDa band) of MMP-2 in the normal aorta $(\mathrm{n}=7)$, small TAA $(4.0-4.9 \mathrm{~cm} ; \mathrm{n}=8)$, and large TAA $(6.5-8.0 \mathrm{~cm} ; \mathrm{n}=8)$ groups. (Bottom) Densitometric quantitation of active (64-kDa band) MMP-2 protein abundance determined by gelatin zymography. Results are expressed as mean percentage change of normal aorta. Individual data points in each group overlay box plots. The box defines the 25 th to 75 th interquartile range, and both the median with normal aorta $(100 \% \pm 14 \%)$ (Figure $2, A$ and $B$ ). PKC- $\delta$-specific activation was determined by examining the ratio of phospho-PKC- $\delta$ (activated form) to total PKC- $\delta$. The results show a trend toward increased PKC- $\delta$ activity in small TAAs $(201 \% \pm 67 \%)$ and severely reduced PKC- $\delta$ activity in large TAAs $(6 \% \pm 3 \%$; $P<.05$ vs normal) compared with normal aorta $(100 \% \pm 48 \%)$ (Figure 2, $A$ and $C$ ).

MT1-MMP abundance was examined in isolated primary normal and 4-week TAA mouse aortic fibroblast cell lines. The results were consistent with those for the human clinical TAA specimens, showing elevated MT1-MMP protein abundance with TAA fibroblasts compared with control fibroblasts $(281 \% \pm 15 \%$ vs $100 \% \pm 14 \%$; $P<.05$ vs control) (Figure 3, A). To examine MT1-MMP cellular localization, we transfected both normal and 4-week TAA aortic fibroblast cell lines with MT1-MMPGFP. At steady state in control cells, MT1-MMP-GFP was localized predominantly at the plasma membrane, with some MT1-MMP appearing in endosomal vesicles (Figure 3, B, top). In the 4-week TAA aortic fibroblasts, however, MT1-MMP-GFP was localized almost exclusively in endosomal vesicles (Figure 3, B, bottom).

To examine the regulation of MT1-MMP cellular localization, we treated normal aortic fibroblasts transfected with MT1-MMP-GFP with PMA in the absence or presence of the PKC- $\delta$-specific inhibitor röttlerin. The results show that MT1-MMP-GFP was localized predominantly at the plasma membrane in vehicle-treated cells (control; Figure 4, top left). On stimulation with PMA, MT1-MMP-GFP translocated intracellularly, appearing almost exclusively in endosomal vesicles (PMA; Figure 4, top right). When the cells were pretreated with röttlerin, followed by PMA, MT1-MMP-GFP intracellular translocation was inhibited (röttlerin + PMA; Figure 4, bottom left). In fact, treatment with röttlerin alone appeared to be sufficient to lock MT1-MMP-GFP at the plasma membrane (röttlerin; Figure 4, bottom right).

To assess the effects of MT1-MMP localization on protein function, we treated normal aortic fibroblasts transfected with MT1-MMP-GFP with PMA in the absence or presence of röttlerin. The cells were then collected for biochemical analysis by immunoblotting for activated MMP-2 or evidence of activated TGF- $\beta$ signaling. The results show that normal aortic fibroblast cell lines treated with PMA displayed reduced MMP-2 activation $(73 \% \pm 4 \%$; $P<.05$ vs control) (Figure $5, A$ ) and increased phosphorylation of Smad2 $(257 \% \pm 44 \% ; P<.05$ vs control) (Figure 5,

(red line) and mean (blue line) values are shown within the box. $* P<.05$ versus normal; $\# P<.05$ versus small TAA, ANOVA with Tukey's WSD. TAA, Thoracic aortic aneurysm; MT1-MMP, membrane type-1 matrix metalloproteinase. 


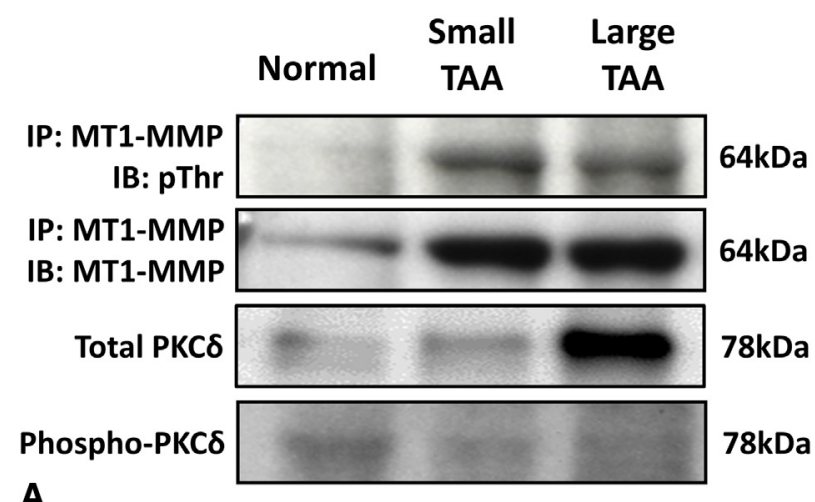

A

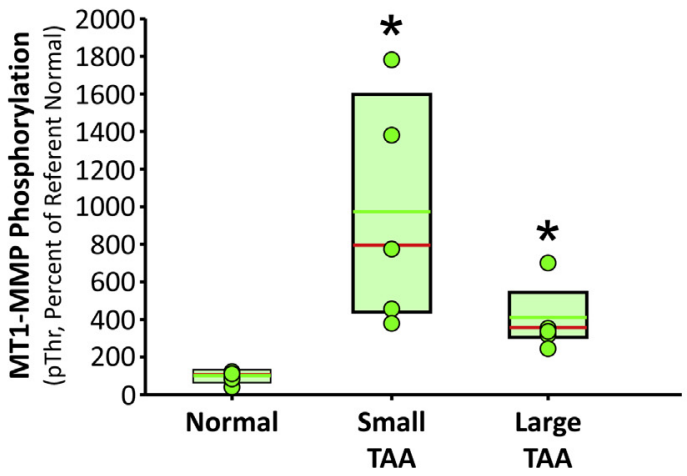

C

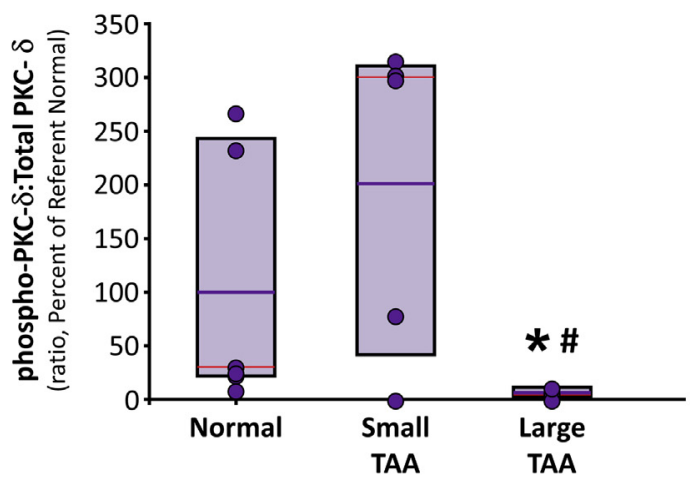

FIGURE 2. Phosphorylation of MT1-MMP in clinical TAA specimens. A, MT1-MMP was immunoprecipitated from the normal aorta, small TAA $(4.0-4.9 \mathrm{~cm})$, and large TAA $(6.5-8.0 \mathrm{~cm})$ groups. The immunoprecipitated protein was fractionated by electrophoresis and immunoblotted for phospho-threonine and MT1-MMP to confirm immunoprecipitation. Tissue homogenates from the same sample set were also immunoblotted for total PKC- $\delta$ and phospho-PKC- $\delta$. Representative images are shown. B, Densitometric quantitation of MT1-MMP-specific phospho-threonine residues in the normal aorta $(\mathrm{n}=6)$, small TAA $(4.0-4.9 \mathrm{~cm} ; \mathrm{n}=5)$, and large TAA $(6.5-8.0 \mathrm{~cm} ; \mathrm{n}=5)$ groups. Results are expressed as mean percentage change of normal aorta. Individual data points in each group overlay box plots. The box defines the 25 th to 75 th interquartile range, and both the median (red line) and mean (green line) values are shown within the box. $* P<.05$ versus normal, ANOVA with Tukey's WSD. C, Densitometric quantitation of the ratio of phospho-PKC- $\delta$ (activated) to total PKC- $\delta$ from the normal aorta $(\mathrm{n}=6)$, small TAA $(4.0-4.9 \mathrm{~cm}$; $\mathrm{n}=5)$, and large TAA $(6.5-8.0 \mathrm{~cm} ; \mathrm{n}=4)$ groups. Results are expressed as mean percentage change of normal aorta. Individual data points in each group overlay box plots. The box defines the 25 th to 75 th interquartile
$B$ ), the intracellular mediator of activated TGF- $\beta$ receptors. Importantly, in both circumstances, these effects were inhibited by pretreatment with röttlerin (MMP-2, $100 \% \pm 4 \%$; pSmad2, $159 \% \pm 51 \%$ ) (Figure $5, A$ and $B$ ).

\section{DISCUSSION}

Aortic medial degeneration is a common feature of TAA, characterized by disruption of the medial elastic lamellae, alterations in collagen deposition, and changes in cellular content/phenotype. Previous studies have identified and characterized a multifunctional membrane-bound protease, MT1-MMP, with several unique characteristics that position it at the center of multiple mechanisms capable of regulating ECM deposition and degradation. Multiple lines of evidence from both human and animal studies have suggested that MT1-MMP plays an essential role in aortic structural remodeling during TAA development. ${ }^{3,6,12,27,28}$ Importantly, because MT1-MMP is activated cotranslationally, defining the mechanisms that govern its intracellular activity and localization during TAA development are essential for understanding its role in pathogenesis. Accordingly, in the present study we hypothesized that MT1-MMP activity and cellular localization are regulated by phosphorylation during TAA formation and progression. To test this hypothesis, we selected 2 cohorts of TAA specimens from an established aortic tissue bank (small TAAs, 4.0-4.9 cm; large TAAs, 6.5-8.0 cm) and analyzed and compared MT1-MMP values with those measured in normal aortic specimens harvested from patients without aneurysm disease.

The significant findings of this study are manifold. First, MT1-MMP protein abundance was elevated in both small and large TAA specimens compared with normal aortas. This increase suggests that MT1-MMP may indeed play an important role in TAA formation and progression. Second, the latent and active forms of MMP-2 were measured, and, interestingly, the activated form was increased only in the large TAA specimens. Because MT1-MMP plays a well-described role as a constitutive mediator of latent-MMP-2 activation, the disparity between increased MT1-MMP in the absence of increased active MMP-2 in small TAA specimens, suggested that MT1-MMP might be post-translationally regulated. Third, the observed increase in MT1-MMP-specific phospho-threonine demonstrates, for the first time, that MT1-MMP is unequivocally differentially phosphorylated

range, and both the median (red line) and mean (purple line) values are shown within the box. $* P<.05$ versus normal; $\# P<.05$ versus small TAA, ANOVA with Tukey's WSD. TAA, Thoracic aortic aneurysm; $M T 1-M M P$, membrane type-1 matrix metalloproteinase; $P K C$, protein kinase $\mathrm{C}$. 

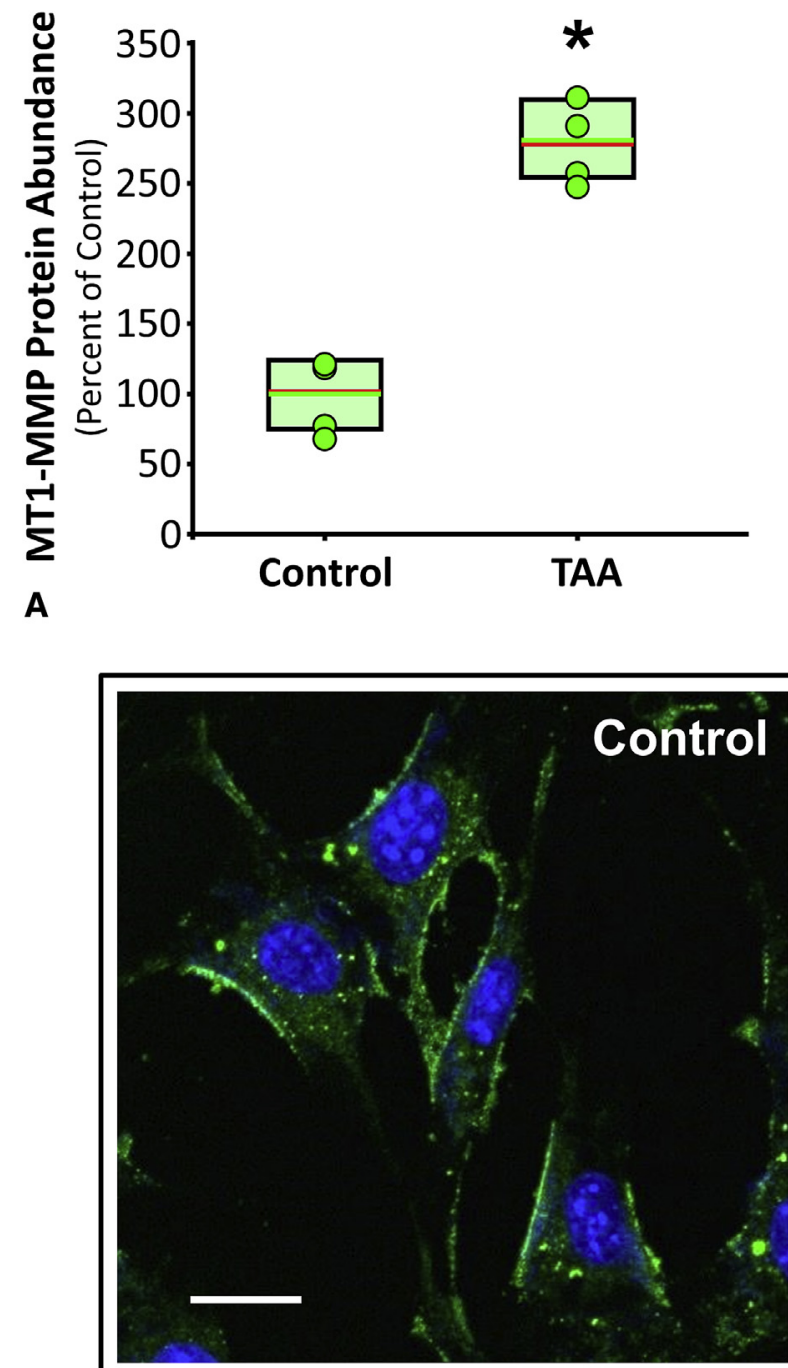

B

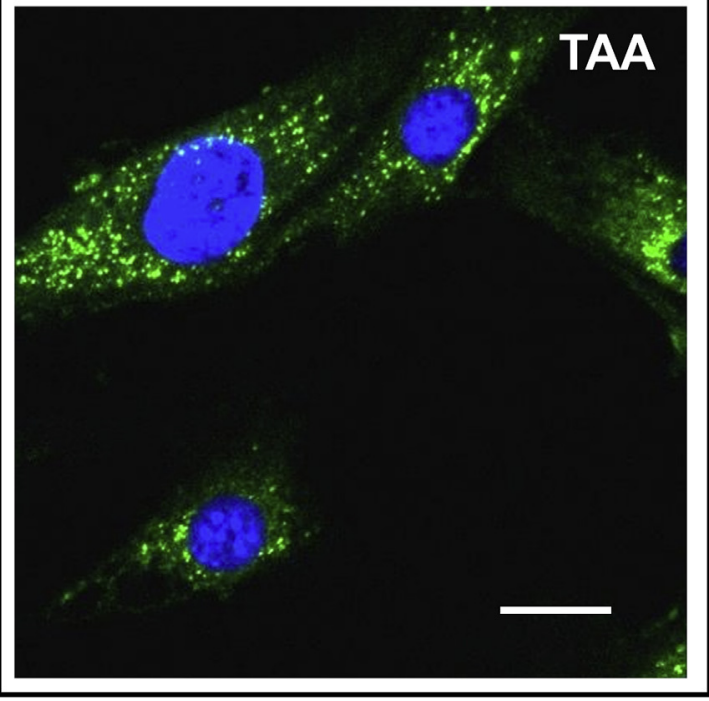

FIGURE 3. MT1-MMP abundance and localization in primary aortic fibroblasts. A, Protein abundance of MT1-MMP in normal and 4-week TAA aortic fibroblasts $(\mathrm{n}=4$ each group). Results are expressed as mean percentage change of control aorta. Individual data points in each during TAA development. Fourth, studies examining primary aortic fibroblast cell lines isolated from normal and 4-week TAA mice transfected with GFP-tagged MT1-MMP demonstrated that MT1-MMP was localized predominantly at the plasma membrane surface in normal aortic fibroblasts, but was localized almost exclusively to intracellular endosomal vesicles in 4-week TAA aortic fibroblasts.

Finally, activation of PKC could induce the translocation of MT1-MMP from the membrane surface into endosomal vesicles, and this process could be inhibited by röttlerin, a PKC- $\delta$-specific pharmacologic inhibitor. Moreover, when MT1-MMP was localized to the plasma membrane (unphosphorylated; steady-state or in the presence of röttlerin), it functioned to activate latent MMP-2, however, when MT1-MMP was internalized in endosomal vesicles (phosphorylated; PMA), it functioned to activate TGF- $\beta$ receptor signaling. The changes in MT1-MMP abundance and activity observed in this study suggest that MT1-MMP may function to promulgate aneurysm progression through multiple pathways. Accordingly, identifying the independent roles of MT1-MMP in small and large TAAs may reveal multiple mechanisms that could be targeted for clinical benefit.

The present study examined MT1-MMP in small and large clinical specimens and found elevated MT1-MMP protein abundance with the advancement of TAA disease, suggesting that MT1-MMP may play an important role in clinical TAA formation and progression. This idea is supported in a recent study reported by Jackson et al, ${ }^{27}$ who identified increased MT1-MMP mRNA expression and medial immunohistochemical staining in clinical TAA specimens associated with both bicuspid and tricuspid aortic valves. Furthermore, previous work from this laboratory, using an established murine model of TAA, demonstrated a positive correlation between MT1-MMP activity and the time-dependent change in aortic diameter over a 16-week time course after TAA induction. ${ }^{3}$

Many studies have examined MT1-MMP activity indirectly; most have used the activation of pro-MMP-2 as a surrogate indicator. Thus, in this study we examined the activation of latent MMP-2. A sharp increase in active MMP-2 abundance was observed in large TAAs. These

group overlay box plots. The box defines the 25 th to 75 th interquartile range, and both the median (red line) and mean (green line) values are shown within the box. $* P<.05$ versus control, unpaired Student's $t$ test. $\mathrm{B}$, Representative images from normal and 4-week TAA aortic fibroblasts transfected with MT1-MMP-GFP. At steady state, MT1-MMP-GFP was localized predominantly at the plasma membrane in the normal cells, but was almost exclusively localized in endosomal vesicles in the 4-week TAA cells. TAA, Thoracic aortic aneurysm; MT1-MMP, membrane type-1 matrix metalloproteinase. 
results suggest that increased MT1-MMP activity serves to amplify and accelerate the proteolytic response by activating MMP-2. These results are consistent with previous animal studies in abdominal aortic aneurysms demonstrating that the MT1-MMP-dependent activation of MMP-2 is essential for aneurysm development. ${ }^{28,29}$ Moreover, studies by Xiong et $\mathrm{al}^{30}$ demonstrated that aortic dilatation in abdominal aortic aneurysms was inhibited in a TIMP-2-deficient mouse. Although this finding was initially contrary to the expected result for the removal of an MMP inhibitor, the investigators demonstrated that the loss of TIMP-2 prevented the MT1-MMP-dependent activation of MMP-2, which is necessary for aneurysm formation. Interestingly, in the present study we found a disparity between elevated MT1-MMP and active MMP-2 in small TAAs. This finding is consistent with a previous study from our laboratory that found a similar disparity between MT1-MMP and MMP-2 activation during TAA formation in an established murine model. ${ }^{3}$ This suggests that either the low level of MT1-MMP activity is sufficient to carry out specific activities independent of MMP-2 activation, or MT1-MMP is post-translationally regulated.

As a type I transmembrane protein, MT1-MMP is likely subject to regulatory mechanisms that direct cellular localization through the endosomal-plasma membrane recycling process. As a result, endocytic recycling of MT1-MMP is one mechanism by which access to specific enzymatic substrates could be controlled. Previous work
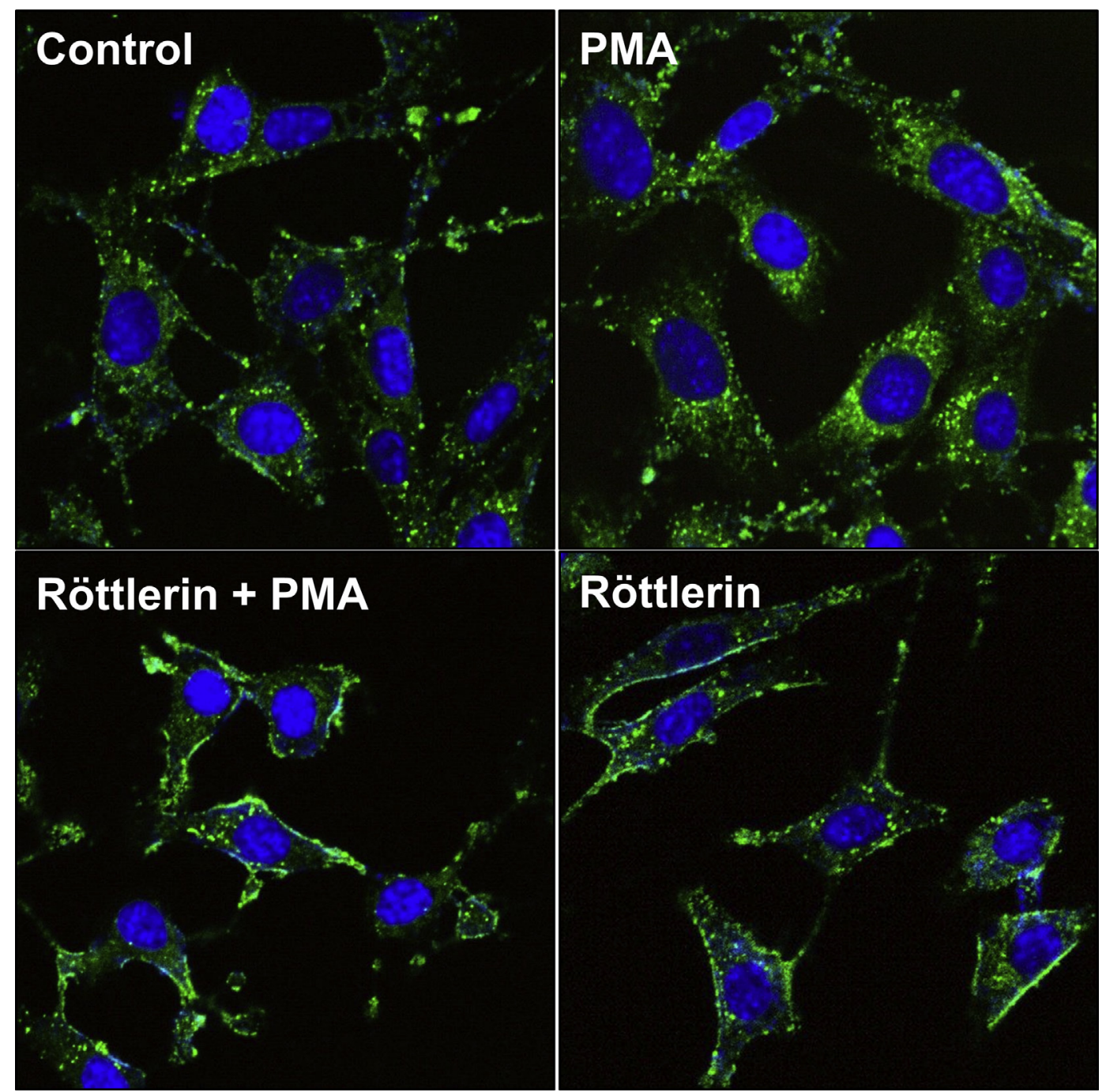

FIGURE 4. PKC- $\delta$-mediated MT1-MMP translocation in normal aortic fibroblasts. Representative images of primary aortic fibroblasts transfected with MT1-MMP-GFP and treated with or without PMA, in the presence or absence of röttlerin. Images show MT1-MMP-GFP predominantly at the plasma membrane in controls. On activation of PKC with PMA, MT1-MMP translocated to intracellular endosomal vesicles. When the cells were pretreated with the PKC- $\delta$-specific inhibitor röttlerin, PMA-induced translocation was inhibited. röttlerin alone was sufficient to lock MT1-MMP at the plasma membrane. PMA, Phorbol 12-myristate 13-acetate. 

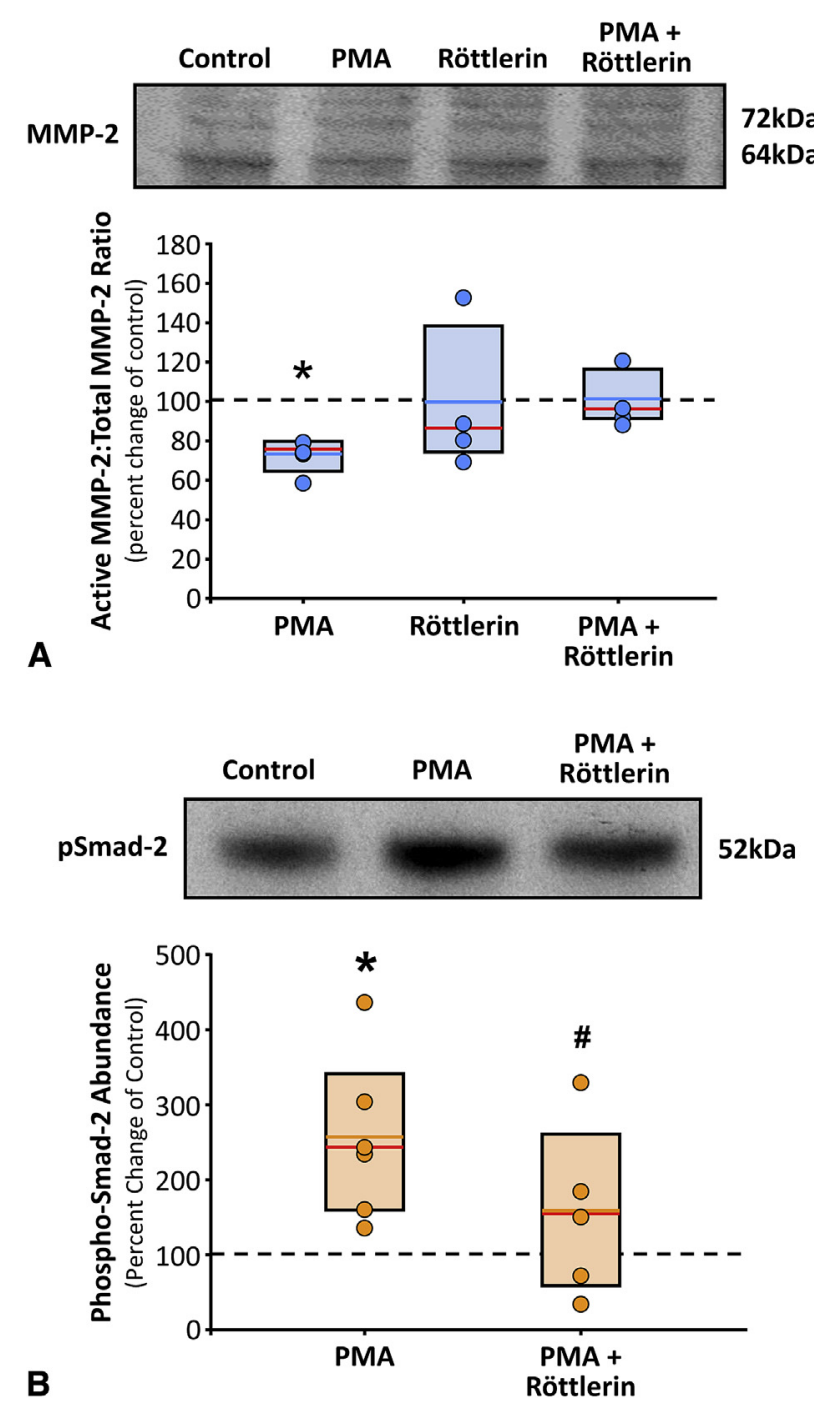

FIGURE 5. PMA-induced changes in MT1-MMP function. A, Primary aortic fibroblasts from normal mice $(n=4)$ were treated with or without PMA, in the presence or absence of röttlerin for $18 \mathrm{~h}(\mathrm{n}=4$ each treatment group). Cell homogenates were fractionated by gel electrophoresis and immunoblotted for latent and active MMP2. Results are expressed as mean percentage change in each cell line from its respective steady-state control (vehicle-treated; dotted line). Individual data points in each group overlay box plots. The box defines the 25 th to 75 th interquartile range, and both the median (red line) and mean (blue line) values are shown within the box. $* P<.05$ versus control, ANOVA with Tukey's WSD. B, Primary aortic fibroblasts from normal mice $(n=4)$ were treated with or without PMA (30 minutes), in the presence or absence of röttlerin (3 hours of preincubation, plus 30 minutes concurrent; $\mathrm{n}=4$ for each treatment group). Cell homogenates were fractionated by gel electrophoresis and immunoblotted for phospho-Smad2. Results are expressed as mean percentage change in each cell line from its respective steady-state control (vehicle-treated; dotted line). Individual data points in each group overlay box plots. The box defines the 25 th to 75 th interquartile range, and both the median (red line) and mean (orange line) values are shown within the box. ${ }^{*} P<.05$ versus control; $\# P<.05$ versus PMA, ANOVA with Tukey's WSD. PMA, Phorbol 12-myristate 13-acetate; MMP, matrix metalloproteinase. has shown that MT1-MMP requires a functional transmembrane domain to be correctly localized for latent MMP-2 activation. ${ }^{20}$ Similarly, it was demonstrated that the cytoplasmic tail of MT1-MMP is required for endocytosis and trafficking along the microtubular network. $^{22,23}$ To explore potential posttranslational modifications of MT1-MMP that could affect endosomal recycling, we used in silico bioinformatics to examine the C-terminal amino acid sequence for regulatory sites. Interestingly, we identified threonine-567 (T567) as a potential PKC-mediated phosphorylation site localized within the short 20-amino acid cytoplasmic tail of MT1-MMP. Indeed, several studies have established that MT1-MMP activity and/or cellular localization can be regulated by phosphorylation of C-terminal residues. ${ }^{22,24,25}$ Williams et $\mathrm{al}^{25}$ demonstrated that PKC-dependent phosphorylation of T567 leads to enhanced internalization of MT1-MMP. Moss et $\mathrm{al}^{24}$ reported that T567 phosphorylation resulted in altered substrate specificity and cellular localization, and suggested that PKC- $\delta$ may be the kinase responsible. In the present study, immunoprecipitation of MT1-MMP, followed by immunoblotting for phosphothreonine residues, demonstrated increased MT1-MMP phospho-threonine phosphorylation in small and large TAAs. This coincided with increased PKC- $\delta$ activation in small TAAs and with a dramatic reduction in activated PKC- $\delta$ in large TAAs.

To examine the functional consequences of MT1-MMP phosphorylation, we expressed GFP-tagged MT1-MMP in primary aortic fibroblasts isolated from normal and 4-week TAA mice. Interestingly, at steady state in the absence of any treatment, MT1-MMP was localized predominantly at the plasma membrane, with some in endosomes, in the control cells, but was localized almost exclusively in endosomal vesicles in the TAA-derived cells. This suggests that the internalization of MT1-MMP likely plays an important role in mediating the cellular changes occurring during TAA development.

Normal murine aortic fibroblasts were treated with an activator of PKC in the presence and absence of the PKC- $\delta$-specific inhibitor röttlerin. These studies demonstrated that inducing phosphorylation of MT1-MMP through the activation of PKC- $\delta$, could indeed induce the translocation of MT1-MMP-GFP from the plasma membrane into endosomal vesicles, thereby altering its access to specific MT1-MMP substrates. This suggests that the reduced activation of MMP-2 in the small clinical TAAs may be the result of enhanced internalization of MT1-MMP. Furthermore, TGF- $\beta$ is known to be commonly sequestered within the ECM, bound by latent TGF- $\beta$-binding proteins and localized to the fibrillin microfibrils. Both latent TGF- $\beta$-binding protein 1 and fibrillin 1a are vital components of the elastic microarchitecture within the aortic wall, and have been identified as 


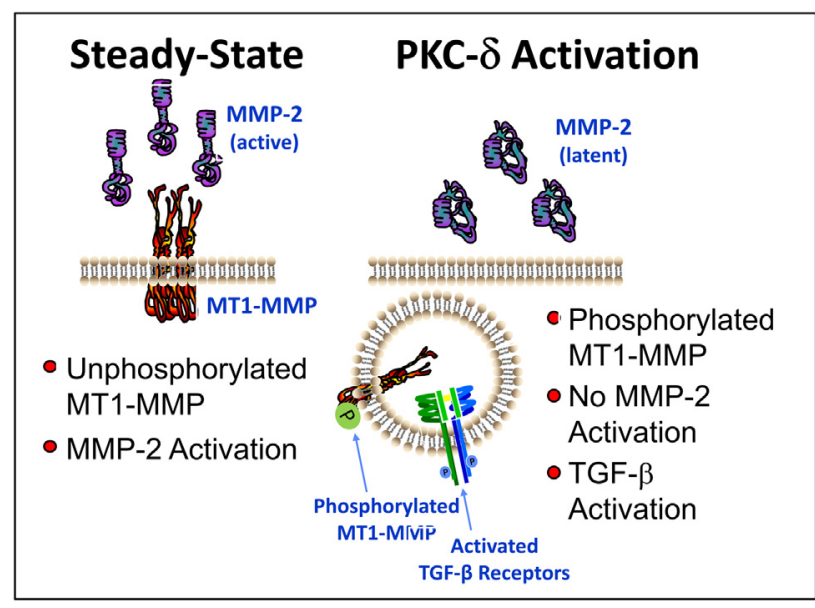

FIGURE 6. At steady-state (left), membrane type-1 matrix metalloproteinase $(M T 1-M M P)$ remains unphosphorylated and is localized to the plasma membrane surface where it functions to activate latent-MMP-2. On cellular stimulation and activation of protein kinase $\mathrm{C}(P K C)-\delta$ (right), MT1-MMP is phosphorylated on its $\mathrm{C}$-terminus, and is internalized in endosomal vesicles. Internalization is associated with the phosphorylation of Smad-2, a downstream intermediate activated by transforming growth factor $(T G F)$ $\beta$ receptor signaling. Together this suggests that MT1-MMP activity is regulated by altering its cellular localization.

substrates for MT1-MMP. ${ }^{31,32}$ Importantly, enhanced TGF- $\beta$ signaling has been implicated in aneurysm development through multiple mechanisms, including the dysregulation of collagen deposition, enhanced production of ECM-degrading enzymes, and induction of changes in cellular composition and phenotype. ${ }^{33}$ Accordingly, we then examined MT1-MMP phosphorylation in relationship to the release of sequestered TGF- $\beta$ and activation of the TGF- $\beta$ signaling pathway, as determined by the phosphorylation of Smad2. Remarkably, when cells were treated with the activator PKC- $\delta$, a concomitant increase in phospho-Smad2 was observed, in a PKC- $\delta$-dependent fashion (results are summarized in Figure 6). These results are consistent with previous observations that TGF- $\beta$ signaling may be enhanced and prolonged on internalization of the TGF- $\beta$ receptor complex. ${ }^{34,35}$

Although our results suggest a multifunctional role for MT1-MMP in TAA development, this study is not without some limitations. First, it must be recognized that the ascending TAA specimens were selected based on aortic size alone. Accordingly, because all aortic specimens were collected when deemed surgically necessary, the clinical indications leading to early surgical intervention may have resulted in the selection of more aggressive TAAs in the small cohort. Second, because of significant differences in patient age among the normal, small TAA, and large TAA cohorts, the possibility of age-dependent effects on MT1-MMP abundance and activity cannot be ruled out. Finally, although the cellular localization of
MT1-MMP could not be determined in the clinical TAA specimens, it must be recognized that there are distinct differences compared with cells isolated from the murine TAA model, with respect to time and progression of TAA disease. The difficulty in obtaining human aortic fibroblasts from patients without aortic disease prevented the completion of these studies using cells/tissue solely of human origin.

Nevertheless, our unique findings in this study demonstrate that MT1-MMP is elevated in clinical TAA specimens, and that phosphorylation of MT1-MMP regulates enzymatic function by altering its cellular localization, mediating access to specific substrates and shifting its functional role from MMP-2 activation to mediator of intracellular signaling. Although this study cannot differentiate the importance of the location-specific activity of MT1-MMP in relationship to advancing TAA development, our results do suggest that targeting MT1-MMP activity or protein abundance may provide a novel therapeutic strategy for treating TAA disease.

\section{Conflict of Interest Statement}

Authors have nothing to disclose with regard to commercial support.

\section{References}

1. Elefteriades JA. Natural history of thoracic aortic aneurysms: indications for surgery, and surgical versus nonsurgical risks. Ann Thorac Surg. 2002;74: S1877-80; discussion S1892-8.

2. Barbour JR, Spinale FG, Ikonomidis JS. Proteinase systems and thoracic aortic aneurysm progression. J Surg Res. 2007;139:292-307.

3. Jones JA, Ruddy JM, Bouges S, Zavadzkas JA, Brinsa TA, Stroud RE, et al. Alterations in membrane type-1 matrix metalloproteinase abundance after the induction of thoracic aortic aneurysm in a murine model. Am J Physiol Heart Circ Physiol. 2010;299:H114-24.

4. Longo GM, Xiong W, Greiner TC, Zhao Y, Fiotti N, Baxter BT. Matrix metalloproteinases 2 and 9 work in concert to produce aortic aneurysms. J Clin Invest. 2002;110:625-32.

5. Shen M, Lee J, Basu R, Sakamuri SS, Wang X, Fan D, et al. Divergent roles of matrix metalloproteinase 2 in pathogenesis of thoracic aortic aneurysm. Arterioscler Thromb Vasc Biol. 2015;35:888-98.

6. Xiong W, Knispel R, MacTaggart J, Greiner TC, Weiss SJ, Baxter BT. Membrane-type 1 matrix metalloproteinase regulates macrophage-dependent elastolytic activity and aneurysm formation in vivo. J Biol Chem. 2009;284 1765-71.

7. Habashi JP, Judge DP, Holm TM, Cohn RD, Loeys BL, Cooper TK, et al. Losartan, an AT1 antagonist, prevents aortic aneurysm in a mouse model of Marfan syndrome. Science. 2006;312:117-21.

8. Jones JA, Barbour JR, Stroud RE, Bouges S, Stephens SL, Spinale FG, et al. Altered transforming growth factor-beta signaling in a murine model of thoracic aortic aneurysm. J Vasc Res. 2008;45:457-68.

9. Jones JA, Spinale FG, Ikonomidis JS. Transforming growth factor-beta signaling in thoracic aortic aneurysm development: a paradox in pathogenesis. J Vasc Res. 2009;46:119-37.

10. Pyeritz RE. Recent progress in understanding the natural and clinical histories of the Marfan syndrome. Trends Cardiovasc Med. 2016;26:423-8.

11. Annabi B, Shédid D, Ghosn P, Kenigsberg RL, Desrosiers RR, Bojanowski MW et al. Differential regulation of matrix metalloproteinase activities in abdominal aortic aneurysms. J Vasc Surg. 2002;35:539-46.

12. Ikonomidis JS, Jones JA, Barbour JR, Stroud RE, Clark LL, Kaplan BS, et al. Expression of matrix metalloproteinases and endogenous inhibitors within 
ascending aortic aneurysms of patients with Marfan syndrome. Circulation. 2006;114(1 Suppl):I365-70

13. Komiyama T, Coppola JM, Larsen MJ, van Dort ME, Ross BD, Day R, et al. Inhibition of furin/proprotein convertase-catalyzed surface and intracellular processing by small molecules. J Biol Chem. 2009;284:15729-38.

14. Itoh Y, Seiki M. MT1-MMP: a potent modifier of pericellular microenvironment J Cell Physiol. 2006;206:1-8.

15. Imai K, Ohuchi E, Aoki T, Nomura H, Fujii Y, Sato H, et al. Membrane-type matrix metalloproteinase 1 is a gelatinolytic enzyme and is secreted in a complex with tissue inhibitor of metalloproteinases 2. Cancer Res. 1996;56: 2707-10.

16. Ohuchi E, Imai K, Fujii Y, Sato H, Seiki M, Okada Y. Membrane type 1 matrix metalloproteinase digests interstitial collagens and other extracellular matrix macromolecules. J Biol Chem. 1997;272:2446-51.

17. Deryugina EI, Ratnikov B, Monosov E, Postnova TI, DiScipio R, Smith JW, et al MT1-MMP initiates activation of pro-MMP-2 and integrin alphavbeta3 promotes maturation of MMP-2 in breast carcinoma cells. Exp Cell Res. 2001; 263:209-23

18. Knäuper V, Will H, López-Otin C, Smith B, Atkinson SJ, Stanton H, et al Cellular mechanisms for human procollagenase-3 (MMP-13) activation. Evidence that MT1-MMP (MMP-14) and gelatinase a (MMP-2) are able to generate active enzyme. J Biol Chem. 1996;271:17124-31.

19. Mu D, Cambier S, Fjellbirkeland L, Baron JL, Munger JS, Kawakatsu H, et al The integrin alpha(v)beta8 mediates epithelial homeostasis through MT1-MMP-dependent activation of TGF-beta1. J Cell Biol. 2002;157:493-507.

20. Cao J, Sato H, Takino T, Seiki M. The C-terminal region of membrane type matrix metalloproteinase is a functional transmembrane domain required for pro-gelatinase A activation. J Biol Chem. 1995;270:801-5.

21. Jiang A, Lehti K, Wang X, Weiss SJ, Keski-Oja J, Pei D. Regulation of membrane-type matrix metalloproteinase 1 activity by dynamin-mediated endocytosis. Proc Natl Acad Sci U S A. 2001;98:13693-8.

22. Remacle AG, Rozanov DV, Baciu PC, Chekanov AV, Golubkov VS, Strongin AY The transmembrane domain is essential for the microtubular trafficking of membrane type-1 matrix metalloproteinase (MT1-MMP). J Cell Sci. 2005; 118(Pt 21):4975-84.

23. Uekita T, Itoh Y, Yana I, Ohno H, Seiki M. Cytoplasmic tail-dependent internalization of membrane-type 1 matrix metalloproteinase is important for its invasion-promoting activity. J Cell Biol. 2001;155:1345-56.

24. Moss NM, Wu YI, Liu Y, Munshi HG, Stack MS. Modulation of the membrane type 1 matrix metalloproteinase cytoplasmic tail enhances tumor cell invasion and proliferation in three-dimensional collagen matrices. J Biol Chem. 2009;284:19791-9.
25. Williams KC, Coppolino MG. Phosphorylation of membrane type 1-matrix metalloproteinase (MT1-MMP) and its vesicle-associated membrane protein 7 (VAMP7)-dependent trafficking facilitate cell invasion and migration. J Biol Chem. 2011;286:43405-16.

26. Jones JA, Zavadzkas JA, Chang EI, Sheats N, Koval C, Stroud RE, et al. Cellular phenotype transformation occurs during thoracic aortic aneurysm development. J Thorac Cardiovasc Surg. 2010;140:653-9.

27. Jackson V, Olsson T, Kurtovic S, Folkersen L, Paloschi V, Wågsäter D, et al Matrix metalloproteinase 14 and 19 expression is associated with thoracic aortic aneurysms. J Thorac Cardiovasc Surg. 2012;144:459-66.

28. Sinha I, Hannawa KK, Eliason JL, Ailawadi G, Deogracias MP, Bethi S, et al. Early MT-1 MMP expression following elastase exposure is associated with increased cleaved MMP-2 activity in experimental rodent aortic aneurysms. Surgery. 2004; 136:176-82.

29. Eagleton MJ, Ballard N, Lynch E, Srivastava SD, Upchurch GR Jr, Stanley JC Early increased MT1-MMP expression and late MMP-2 and MMP-9 activity during Angiotensin II induced aneurysm formation. J Surg Res. 2006; $135: 345-51$

30. Xiong W, Knispel R, Mactaggart J, Baxter BT. Effects of tissue inhibitor of metalloproteinase 2 deficiency on aneurysm formation. J Vasc Surg. 2006;44: 1061-6.

31. Ashworth JL, Murphy G, Rock MJ, Sherratt MJ, Shapiro SD, Shutterworth CA, et al. Fibrillin degradation by matrix metalloproteinases: implications for connective tissue remodelling. Biochem J. 1999;340(Pt 1):171-81.

32. Tatti O, Vehviläinen $\mathrm{P}$, Lehti K, Keski-Oja J. MT1-MMP releases latent TGF-beta1 from endothelial cell extracellular matrix via proteolytic processing of LTBP-1. Exp Cell Res. 2008;314:2501-14.

33. Jones JA, Beck C, Barbour JR, Zavadzkas JA, Mukherjee R, Spinale FG, et al Alterations in aortic cellular constituents during thoracic aortic aneurysm development: myofibroblast-mediated vascular remodeling. Am J Pathol. 2009; $175: 1746-56$

34. Hayes S, Chawla A, Corvera S. TGF beta receptor internalization into EEA1-enriched early endosomes: role in signaling to Smad2. J Cell Biol. 2002;158:1239-49.

35. Sigismund S, Confalonieri S, Ciliberto A, Polo S, Scita G, Di Fiore PP Endocytosis and signaling: cell logistics shape the eukaryotic cell plan. Physiol Rev. 2012;92:273-366.

Key Words: aneurysm, MT1-MMP, protein kinase C, remodeling, thoracic aorta 